\title{
Technical Transformation and Innovation on Refinery Acid Gas Dry Process Acid Plant
}

\author{
Du Lang, You Min \\ Sinopec Jingmen Branch, Jingmen, China \\ Email address: \\ dul.jmsh@sinopec.com (Du Lang), youm.jmsh@sinopec.com (You Min)

\section{To cite this article:} \\ Du Lang, You Min. Technical Transformation and Innovation on Refinery Acid Gas Dry Process Acid Plant. American Journal of Chemical \\ Engineering. Vol. x, No. x, 2020, pp. x-x. doi: 10.11648/j.ajche.20200804.12
}

Received: August 18, 2020; Accepted: August 31, 2020; Published: September 21, 2020

\begin{abstract}
This article introduces a new and efficient process and device for acid gas recovery in refining and chemical enterprises-hydrogen sulfide dry acid production process and device. The main problems and failures in the operation of the device are explained, and the related problems and the causes of the failures are analyzed in a targeted manner. In the technical transformation of the base and lining of the acid gas incinerator and the adaptive transformation of the electric defogging system, the development and application of new all-glass steel cooling tower, the implementation of new technologies to improve the efficiency of defogging and acid separation, and the mass transfer and heat transfer efficiency of the process medium, The application of "three units" technology to improve the technical and economic level of the device, the use of sodium-alkali neutralization process to treat the wastewater and tail gas of the device, have unique insights and successful experience, and continue to carry out technical improvement, innovation, integration and application of results for the device. The safety, stability, long-term, economic operation and design and improvement of related fields have important guidance and reference significance.
\end{abstract}

Keywords: Sulfuric Acid Plant, Acid Gas, Dry Process Acid Production, Technical Transformation

\section{Introduction}

In the process of petroleum refining and processing, a large number of sulfur-containing process gases are produced. These gases are harmful to the environment. The sulfur element in the gas must be recovered before being discharged after treatment. Generally, The sulfur recovery process is usually used for recovery. This paper introduces the application of a new and High-efficient acid gas recovery method in Refinery, i.e. hydrogen sulfide dry process acid making process. There are some prominent problems in the actual production process, which affect the safe, stable and economic operation of the device [1]. The sulfuric acid plant of Sinopec Jinmen Petrochemical Company, which adopts the acid gas dry acid production process of the refinery. The original design production capacity was $50 \mathrm{kt} / \mathrm{a}$ (founded in 2003). It was put into operation in July 2004 and expanded to $65 \mathrm{kt} / \mathrm{a}$ in 2008. The highest yield of $\mathrm{W}\left(\mathrm{H}_{2} \mathrm{SO}_{4}\right) 98.3 \%$ sulfuric acid produced by the device is $62 \mathrm{kt} / \mathrm{a}$.
The device adopts three-stage reaction and one-stage reaction, two-stage conversion and two-stage absorption dry acid production process. The process flow consists of 3 processes:

1) In the incineration purification and cooling section, Incinerate hydrogen sulfide, the main component of acid gas, into sulfur dioxide, and recover the waste heat to produce $3.5 \mathrm{MPa}$ steam. The carbon dioxide and moisture in the process gas are removed by purification and cooling;

2) In the conversion process, sulfur dioxide is converted into sulfur trioxide under the action of vanadium catalyst

3) In the dry absorption process, sulfuric acid with a mass fraction of $92.5 \%$ to $93.5 \%$ is used to absorb the moisture in the process gas and sulfuric acid with a mass fraction of $98.3 \%$ to $98.5 \%$ is used to absorb sulfur trioxide to produce finished sulfuric acid.

The process flow is shown in Figure 1. 


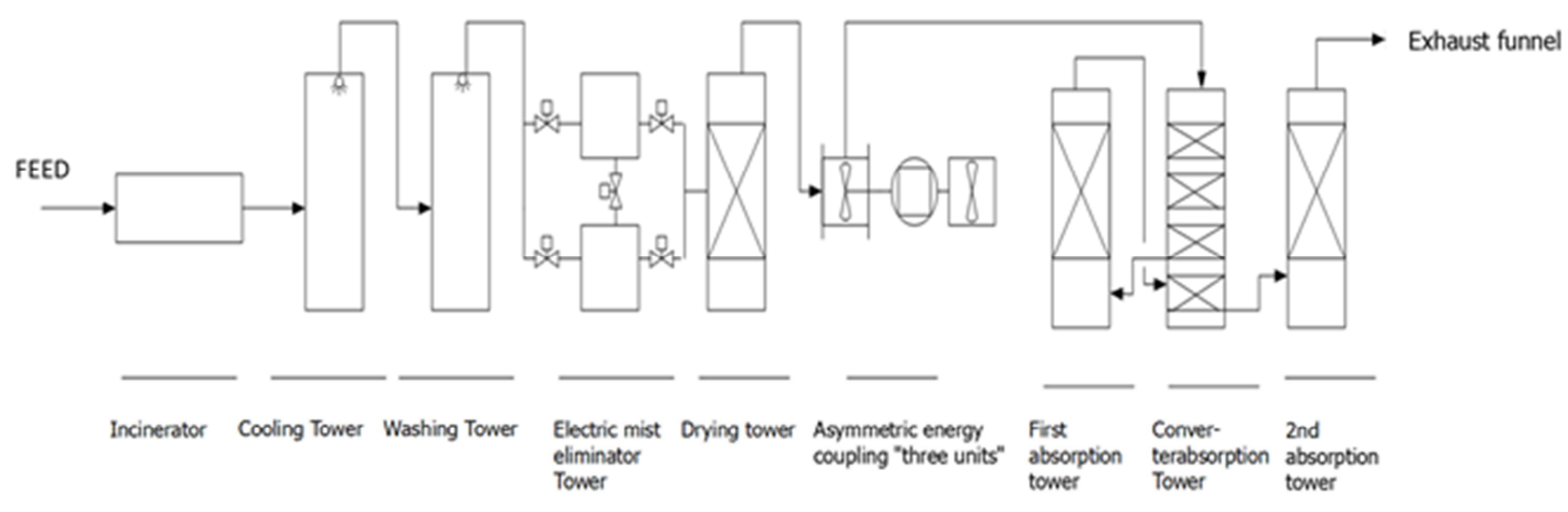

Figure 1. The process flow of acid gas dry process acid plant.

Until 2008, the device has been in the abnormal state of start-up, stop and repair. Every quarter, the plant will be shut down for many times due to its own reasons, and the start-up and shutdown of the device are accompanied by major safety and environmental protection risks.

\section{Problem}

\subsection{The Key Equipment of the Installation Has Major Safety Defects}

For example, the flower wall and the furnace lining in the horizontal high-temperature cylindrical furnace frequently collapse, and the over-temperature of the outer wall causes the shell dew point corrosion and thermal stress compensation problems. The cooling tower and its inlet pipeline are repeatedly perforated due to corrosion. The pipes and industrial pumps used in the sulfuric acid plant are not resistant to corrosion under working conditions, resulting in shortened life cycles.

\subsection{The Equipment and Pipelines of the Heat Exchange System Are Blocked by Acid Mud and Corroded Perforated [2]}

For example, the fourth heat exchanger is blocked by acid sludge and affects the heat exchange effect; the dilute acid pipeline is easy to corrode and perforate, which will cause the device to shut down.

\subsection{Unscientific Use of Energy in Sulfuric Acid Plant and Lack of Process Adjustment Methods}

The heat energy generated during the incineration of hydrogen sulfide is directly discharged to the $1.0 \mathrm{MPa}$ steam system of public works after the waste heat recovery system generates $3.5 \mathrm{MPa}$ steam to reduce temperature and pressure, which causes energy loss.

\subsection{There Is a Risk of Violations of Laws and Regulations in the Wastewater and Exhaust Gas of the Sulfuric Acid Plant}

More stringent emission standards will be implemented for the discharged waste water and tail gas. The existing technology is difficult to meet the new national environmental protection requirements and must be improved.

\section{Solution}

\subsection{Technical Transformation of Horizontal High Temperature Cylindrical Furnace}

\subsubsection{Adaptive Transformation of Furnace Structure}

According to the actual needs of production, the upper limit of the temperature resistance of the original furnace design was increased from $1350^{\circ} \mathrm{C}$ to $1450^{\circ} \mathrm{C}$, and the inner lining was increased from the original 2 layers (heat resistance + heat insulation) to 3 layers (heat resistance + heat insulation + anticorrosive coating). The flower wall in the middle part is bounded by linings of different performance materials at the front and rear ends of the furnace, which solves the problems of frequent collapse of the original flower wall and furnace lining and dew point corrosion of the shell caused by overheating of the outer wall [3].

\subsubsection{Foundation and Lining Modification}

The original design of the 7 fixed saddles was changed to a fixed tail support, and the remaining supports were axially freely movable to solve the problem of different expansion and contraction in different axial positions and different temperature conditions of the incinerator body. Problems such as the correlation between the quantities, reduce the risk of its intrinsic safety, and improve the reliability of the key equipment of the device.[4].

The model of the original thermal insulation material is QA-212B1, and the upgraded model is QA-212Y (its advantages are good thermal insulation and strong corrosion resistance); before pouring the thermal insulation lining, coat the inner wall of the incinerator metal shell Brush a layer of $2 \mathrm{~mm}$ thick anti-dew point corrosion paint to strengthen the dew point corrosion resistance of the outer wall. 
The furnace lining at the front end of the furnace is upgraded on the basis of the original high-temperature refractory prefabricated brick (TA-218B), the new model is TA-218BY; the back-end furnace lining refractory brick uses upgraded high-strength refractory brick (TA-217Y), according to the design drawings Burning shaped (tenon and groove structure) bricks are used as refractory materials to ensure the strength of refractory materials and reduce the possibility of crack leakage, and solve the problems of frequent collapse of the original flower wall and furnace lining and the shell dew point corrosion caused by overheating of the outer wall.
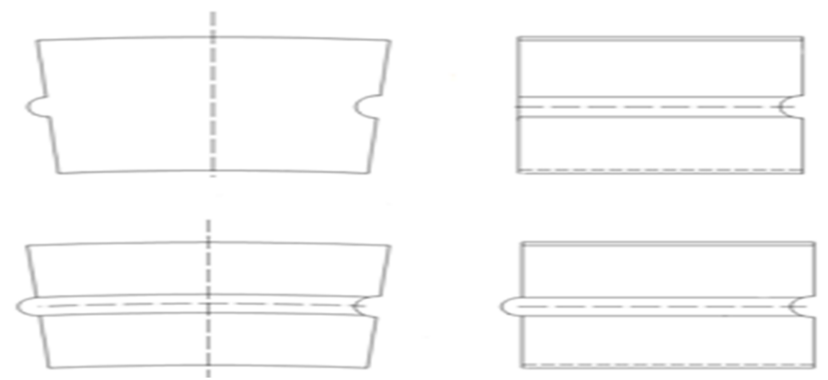

Figure 2. Tenon and groove structure refractory brick.

\subsection{Develop and Apply a New Type of All-glass Steel Cooling Tower}

The cooling tower is the key equipment of the dry sulphuric acid plant. Its working principle and function are: the acidic water sprayed from the top of the tower adiabatically evaporates, and it cools the process gas (the main components are sulfur oxides and nitrogen). Oxide, carbon oxide, sulfide and gaseous sulfur, etc.). During this process, the rising high temperature (about $350^{\circ} \mathrm{C}$ ) process gas in the tower transfers mass and heat with the countercurrent acidic water, and dilute acids (components of $\mathrm{SO}_{4}{ }^{2-}, \mathrm{SO}_{3}{ }^{2-}, \mathrm{NO}_{3}{ }^{-}$) are produced, which objectively requires the tower High temperature and dilute acid corrosion resistance.

The original tower body is a steel composite structure, and the structural form from the outside to the inside is carbon steel, glass steel, lead plates and acid-resistant ceramic tiles. Because the melting point of lead $\left(328^{\circ} \mathrm{C}\right)$ is lower than the temperature of the process gas medium $\left(\geq 350^{\circ} \mathrm{C}\right)$, although there are many measures to protect it, the elbow entering the tower is still often corroded and perforated due to the damage of the lead liner, and then the tower is affected. Ontology, thereby endangering production safety.

Through modeling and computer simulation of the mass transfer and heat transfer process in the internal mass field of the tower, it is confirmed that the new imported hightemperature resistant resin FRP material can meet the actual working conditions. Therefore, in cooperation with related manufacturers, a new type of all-glass steel cooling tower was developed.

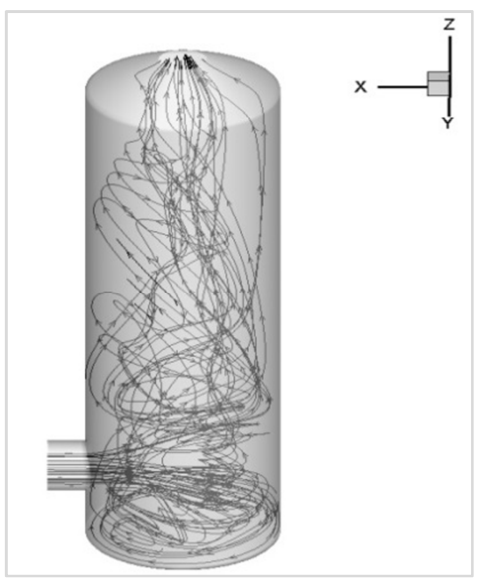

Figure 3. Computer simulation of flue gas flow line diagram.

The tower has a simple structure and a small manufacturing difficulty coefficient. The whole tower is moulded by glass fiber reinforced plastic (shaped steel as ribs): the tower cover is a glass fiber reinforced plastic cone and is integrated with the tower wall; its spray cooling system is a large nozzle connected by a large ring pipe + a metal hose; the entrance elbow (inside) is The carbon steel is lead-lined and lined with special graphite bricks that can withstand both high temperature $\left(\geq 350^{\circ} \mathrm{C}\right)$ and dilute sulfuric acid [5]; the inlet pipeline adopts steel structure lined with glass fiber reinforced plastic, lined with acid-resistant ceramic tiles, and lined with high-alumina graphite brick structure. After thermodynamic calculation, the "U"-shaped bend + bellows + spring support structure is adopted to eliminate the influence of thermal stress and ensure special requirements such as high temperature resistance, dilute acid corrosion resistance and thermal stress reduction of the entrance elbow [6].

\subsection{Adaptation of the Electric Mist Removal System}

Originally designed as a single lead-plastic cylinder electric mist eliminator, its actual defogging ability is poor, and it is easy to form condensed acid and acid mud in the downstream system (equipment and pipeline), corrode and block the downstream system pipeline and equipment.

Modification content: Add a new high-efficiency conductive glass fiber reinforced plastic electric mist eliminator to realize two-stage mist eliminator [7]. In the design of the process flow, the new and old two electric mist eliminators pass through the bypass and the new electric valve (the control signal is remotely transmitted to the DCS room) to realize the free switching between series and parallel. When one of the electric mist eliminators fails It can realize online repair of faulty equipment.

\subsection{Integration of New Technologies in the Drying and Absorption Process}

The main equipment of the dry absorption process is 3 packed towers (namely, drying tower, first suction tower and second suction tower). The structure is shown in Figure 4. 
The principle is to use the packing in the tower to make the process gas fully contact with concentrated sulfuric acid. For mass and heat transfer, the drying tower uses concentrated sulfuric acid to remove water from the process gas, and the absorption tower uses concentrated sulfuric acid to absorb $\mathrm{SO}_{3}$ in the process gas.

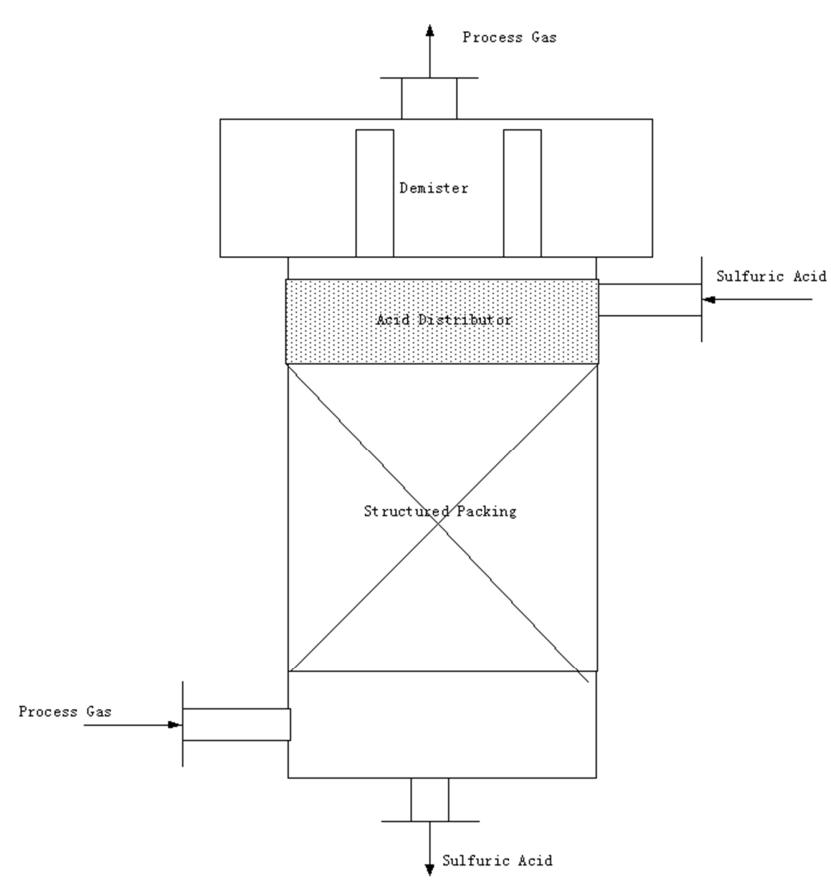

Figure 4. Packing tower structure diagram.

Modification content:

1) The imported candle-type non-wet fiber demister [8] is used to replace the original drawer-type wire mesh demister to improve the demister effect. The original drawer-type wire mesh demister can only remove liquid particles with a particle size greater than $10 \mu \mathrm{m}$, but cannot remove particles below $10 \mu \mathrm{m}$, so that the acid mist can be carried to downstream facilities and equipment after passing through the demister; imported candle-type non-wetting The process of removing liquid particles by the fiber defogger uses Brownian motion as the mechanism of action, which can completely remove the extremely fine acid mist with a particle size of more than $3 \mu \mathrm{m}$, and its defogging efficiency is improved by one level compared with the original drawer-type wire mesh demister;

2) Use external current anode protection, pre-embedded capillary stainless steel tube trough acid distributor, and eliminate the original cast iron tube acid distributor [9]. The original cast iron tubular acid distributor has the key defect of uneven acid distribution caused by the inconsistent degree of corrosion of each acid distribution port and the inconsistent pressure difference between the passages. The new type of pipe-trough acid distributor has the advantages of uniform acid distribution, strong corrosion resistance and clogging ability. The density of acid distribution has been increased from less than 15 units $/ \mathrm{m}^{2}$ of the original cast iron tubular acid distributor to 43 units $/ \mathrm{m}^{2}$;

3) Use high-efficiency porcelain structured packing to eliminate random piled porcelain rings to improve fluid mass and heat transfer efficiency. Compared with traditional random packing, high-efficiency porcelain structured packing has the following advantages:

4) The porosity of the packing is large and the pressure drop is small, which can greatly increase the gas velocity of the empty tower;

5) The geometric structure is reasonable and the specific surface area is moderate, which is conducive to full gasliquid contact, high mass and heat transfer efficiency, and can effectively reduce the height of the packing layer;

6) Wide application range, large operation flexibility, large processing capacity and good stability;

7) The liquid holding capacity is small, which can make the gas and liquid contact evenly in the packing, reduce the phenomenon of effusion, dead angle and wall flow, and will not cause the phenomenon of "liquid flooding".

\subsection{Screening of Pipes and Industrial Pumps That Meet Specific Conditions}

The liquid phase medium of acid gas dry acid production includes dilute sulfuric acid $\mathrm{w}\left(\mathrm{H}_{2} \mathrm{SO}_{4}\right)<15 \%$, concentrated sulfuric acid $\mathrm{W}\left(\mathrm{H}_{2} \mathrm{SO}_{4}\right)>75 \%, 93 \%, 98 \%$, and different processes have different temperature requirements. Pumps and pipes have demanding requirements. [10-12]

About sulfuric acid pipe:

1) The original design of the dilute acid pipeline is made of glass fiber reinforced plastic, but the glass fiber reinforced plastic is very fragile, and it is easily damaged by external force or vibration in case of liquid flow, and it is not suitable for continuous and industrialized production. After comparison and analysis, steel-lined PO (polyolefin) pipes, that is, carbon steel-lined PO, are selected. During manufacturing and installation, the entire pipeline should be divided into multiple small sections (one end of each small section is a fixed flange, and the other end is equipped with a loose flange), and the small section and the small section pass through the flange (fixed flange + loose sleeve) Flange) connection. This not only maintains the rigidity of the carbon steel pipe, but also has good process anti-corrosion performance;

2) Concentrated sulfuric acid pipeline. The temperature range of the tower acid pipeline in the dry absorption process of this device is $80 \sim 110^{\circ} \mathrm{C}$, and the concentration of sulfuric acid $\mathrm{w}\left(\mathrm{H}_{2} \mathrm{SO}_{4}\right)$ is $>75 \%, 93 \%$, and $98 \%$ respectively. It is difficult to flow sulfuric acid under this working condition. A passivation film is formed on the metal surface. The original design material was cast iron, and corrosion and perforation occurred frequently during the actual operation. Now it is changed to molded PTFE pipe material to completely solve the problem. 
About sulfuric acid pump:

1) Dilute acid pump, after fully learning from the failure lessons of the original design selection (inner coating anti-corrosion pump, special stainless steel anticorrosion pump, etc.), successfully screened out the cost-effective (higher) engineering plastic anti-corrosion dilute acid pump;

2) Concentrated acid pump, eliminate the submerged cast iron pump of the original design and selection, and choose the stainless steel concentrated acid pump customized by professional manufacturers.

\subsection{Adopt "Three Units" (Steam Turbine + Motor + Fan) Technology to Improve the Energy Efficiency and Technical and Economic Level of the Device}

The device produces $3.5 \mathrm{MPa}$ steam $(8 \sim 12 \mathrm{t} / \mathrm{h})$. It was originally designed to enter the company's public works
(1.0MPa steam pipe network) system after cooling and reducing pressure. In order to improve the energy efficiency of the operation of the device, "three units" [13] are specially set up, and the structural combination is steam turbine + motor + fan. Due to the limitation of the $3.5 \mathrm{MPa}$ steam produced by the device, the main motor is placed in the middle, and the steam turbine and the main motor jointly drive the fan (the main energy-consuming equipment of the device) in an asymmetrical coupling mode to improve the technical and economic level of the device.

\subsection{A new Wastewater Treatment Process (to Meet the Needs of National Laws and Regulations)}

Wastewater treatment adopts homogeneous, sodium-alkali neutralization treatment process. The process flow is shown in Figure 5:

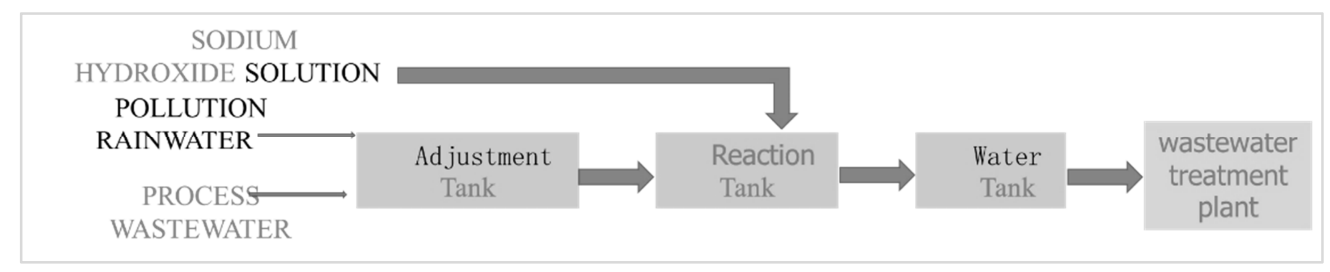

Figure 5. Wastewater treatment process.

\subsection{Newly Added the Process of Tail Gas Treatment with Sodium and Alkali Method Was Added}

Sodium-alkali method is one of the important methods to recover low-concentration sulfur dioxide. Sodium alkali has a strong affinity with sulfur dioxide, high absorption rate, and sodium ions are non-volatile, so there is no secondary pollution [14-16]. The process flow is shown in Figure 6:

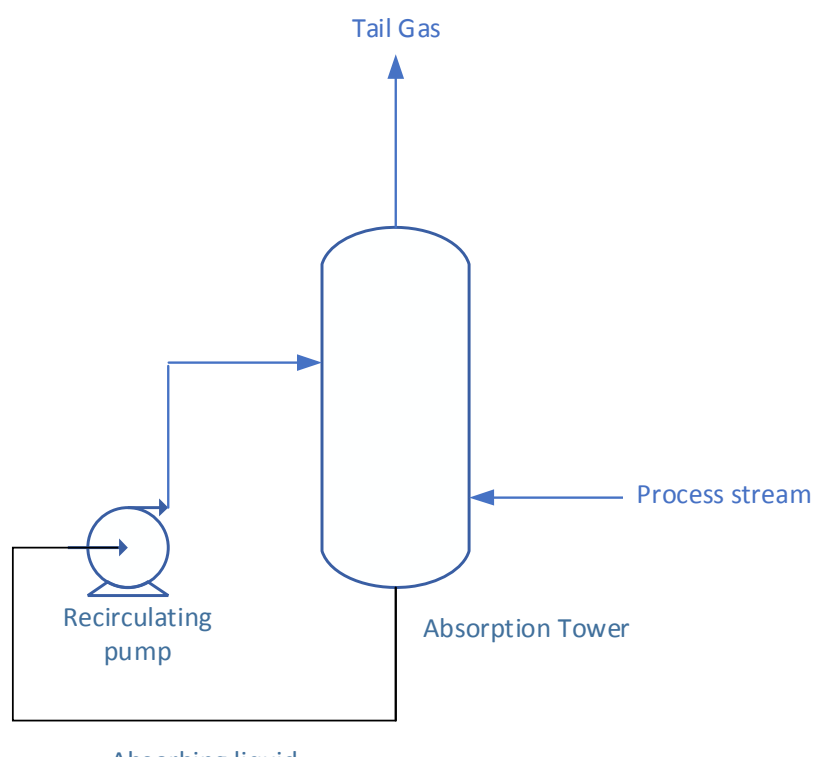

Absorbing liquid

Figure 6. Tail gas treatment process of sodium alkali process.

\section{Implementation Effect}

1) Achieve safe, environmentally friendly, stable and long-term operation of the device. The operation period of the device has been increased from less than one month on average to the current 2-3 years.

2) After a series of optimization measures were completed, the capacity of sulfuric acid plant increased year by year.

3) Through the implementation of "super-large horizontal high-temperature cylindrical furnace technical transformation", it is possible to reduce the risk of hydrogen sulfide incinerator in terms of intrinsic safety and comprehensively improve the reliability of the key equipment of the device. In the design and transformation of super-large horizontal hightemperature cylindrical furnace It has reference value.

4) The "new all-glass steel cooling tower" is adopted to replace the original composite structure tower to reduce equipment purchase cost, installation cost and operation and maintenance cost.

5) The adaptive modification of the electric defogging system, on the one hand, improves the processing capacity and anti-corrosion ability of the electric defogging system. On the other hand, two electric defoggers can be switched in series and parallel freely, which not only improves the flexibility of device operation, but also reduces Plan the risk of shutdown.

6) Asymmetrically set up "three units", greatly improving the energy efficiency of equipment operation. 
7) Scientifically select pipes and industrial pumps that meet specific conditions, and classify and finalize them to achieve long-term operation of sulfuric acid plants.

8) Special treatment of waste water and tail gas generated by the plant is carried out to meet the requirements of national and local laws and regulations on safety and environmental protection and meet the needs of the people in pursuit of a better environment.

\section{Conclusion}

Acid gas drying process directly produces acid gas into sulfuric acid (finished product is sulfuric acid), which makes full use of the chemical energy: generated by sulfur's chemical reaction into sulfuric acid. Compared with the process of producing sulfur (finished product is sulfur) by Claus reaction in refining and chemical enterprises, it has the advantages of more optimized process energy and richer economic benefits, expands the commercial varieties and service scope of enterprises, and opens up a new way for waste acid treatment (waste acid cracking) of sulfuric acid alkylation units in the field.

In this paper, the problems faced by several important links or equipment in the process chain are analyzed from the equipment point of view, and the corresponding solutions are given. All the introduced contents come from the $65 \mathrm{kt} / \mathrm{a}$ acid dry process plant of Jingmen Branch of Sinopec, which is reliable technology and experience verified in production practice and can be replicated and popularized.

The new 150kt/a sour gas dry process (the newly added waste acid cracking process, that is the so-called "two ends and one tail") was successfully started in August 2019, and the introduction of relevant contents can be expected.

\section{References}

[1] CHANG Hai-yang. Construction Design and Material Selection for H2S Burner, Journal of Sulphur Phosphorus \& Bulk Materials Handling Related Engineering, 2018 (04).

[2] Liu Bolong, Cause Analysis of Blocking and Corrosion of Heat Exchanger for Sulphuric Acid Plant and Protection, Journal of Guizhou Chemical Industry, 1998 (3).

[3] GAO Li-cun, JIA Yu-chao et al, Influence of Composite Pore Former on Properties of Corundum-anorthite-mullite Lightweight Castables, Journal of Bulletin of the Chinese Ceramic Society, 2011 (5).

[4] Wu Wenhui, Shi Fei. Structure reconstruction and refractories selection of large horizontal sulphur recovery furnace lining. Journal of Refractories. 2003 (2).

[5] Qin Guozhi. A NOVEL ACID-AND-ALKALI-RESISTANT NONPERMEABLE GRAPHITE AND ITS APPLICATION. Journal of Petrochemical Corrosion and Protection, 1992 (3).

[6] DING Zhenyi. Discussion on Thermal Pipeline Compensation and Common Compensators. Journal of Chemical Equipment Technology, 2018 (6).

[7] ZHANG Jihong, WEI Likai, Failure and maintenance of conductive FRP electrostatic demister, Journal of Sulphuric Acid Industry, 2018 (8).

[8] YE Jianying, ZHOU Shuzhen, Choice and use of mist eliminators of drying and absorption towers in sulphuric acid systems, Journal of Sulphuric Acid Industry, 2004 (1).

[9] WANG Yuhuan, Application of Balanced Pressure and Stabilized Flow Type Acid Distributor in Sulphuric Acid Plant, Journal of Sulphur Phosphorus \& Bulk Materials Handling Related Engineering, 2019 (2).

[10] Luo Fengge, Shao Haiwen, Ni Zhengyu, et al. Development and Application of Corrosion Resistant Cast Alloy for High Temperature Concentrated Sulphuric Acid Pumps, Journal of Sulphuric Acid Industry, 1997 (5).

[11] Yang Jianhong, Failure analysis of engineering plastic pump. Journal of Coastal Enterprises and Science \& Technology, 2011 (6).

[12] Li Yan, Application of Glass Fiber Reinforced Plastic Pipeline in a Large Chemical Plant, Journal of Chemical Engineering Design, 2020 (2).

[13] Zhang Qi. Research on Anti-Surge Control Technology of Three Units of 4.8 Million Tons/Year Catalytic Unit, Journal of Electronic Instrumentation Customers, 2019 (10).

[14] HU Jia. Energy saving and environmental protection design in treating tail gas from sulphuric acid plant of refinery, Journal of Sulphuric Acid Industry, 2017 (04).

[15] LIN Xupeng, LI Maoxian, ZHANG Weibing, Practice and upgrading optimization of the desulphurization system with caustic soda, Journal of Sulphuric Acid Industry, 2018 (7).

[16] WANG Guocai, Application of sodium hydroxide alkali process in the treatment of sulphuric acid tail gas, Journal of Sulphuric Acid Industry, 2018 (4).

\section{Biography}

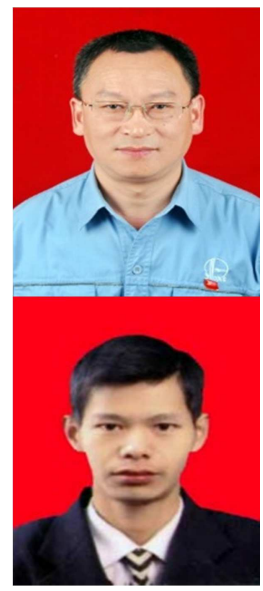

Du Lang, male, born in May 1968, Senior Engineer. Employee of Sinopec Jingmen Branch, engaged in the management of refining and chemical equipment for 31 years.

You Min, male, born in January 1982, Master of Science, Senior Engineer. Employee of Sinopec Jingmen Branch, mainly engaged in environmental protection technology and production operation management. 